\title{
Dynamic Argument Structure
}

\author{
Elisabetta Jezek, Università di Pavia, James Pustejovsky, \\ Brandeis University
}

This paper presents a new classification of verbs of change and modification, proposing a dynamic interpretation of the lexical semantics of the predicate and its arguments. Adopting the model of dynamic event structure proposed in Pustejovsky (2013), and extending the model of dynamic selection outlined in Pustejovsky and Jezek (2011), we define a verb class in terms of its Dynamic Argument Structure (DAS), a representation which encodes how the participants involved in the change behave as the event unfolds. We address how the logical resources and results of change predicates are realized syntactically, if at all, as well as how the exploitation of the resource results in the initiation or termination of a new object, i.e. the result. We show how DAS can be associated with a dynamically encoded event structure representation, which measures the change making reference to a scalar component, modelled in terms of assignment and/or testing of values of attributes of participants.

\section{$1.1 \quad$ Introduction}

In this paper we discuss the patterns in language that are exploited for expressing the semantics of change. We present a classification of change predicates based on a number of syntactic and semantic behaviors involving three semantic parameters: the nature of how the change is measured; the nature of the event capturing the change; and the nature of the change in the participants relative to the event itself.

The kinds of change we will examine in this paper encompass those expressed by both creation and destruction predicates, i.e., predicates that denote the "coming into being" (create and make) and the "going out of being" (disappear and die) of an entity.

The first systematic classification of change, what Aristotle calls 
"generation and corruption" in de Generatione (McKeon, 1968), can be summarized as follows:

(1) Coming into Being:

a. The creation of an object through exploitation of resource:

- natural growth

- aggregation

- manipulation of resource;

b. The creation of something through the representation of a resource.

(2) Going out of Being:

The destruction of an object into existing material

- disaggregation

- termination.

Both creation and destruction verbs have long posed a problem for linguistic classification (see Dowty, 1991, Tenny, 1994, among others) and the interplay between their event structure, and the syntactic expressibility of their arguments is still not well understood (Jezek, 2014).

Creation verbs, for example, exhibit considerable variation in the syntactic realization of the created entity. This is illustrated in the examples below, where we see that, depending on the predicate, the argument role realizing the created object may be: optional (as in 3); mandatory (as in 4); subcategorized as object only (as in 5); as PP only (as in 6); as object or PP (as in 7); or remain hidden as an entailed inference of the sentence (i.e., the resulting photograph in (8)) while still playing a role in the interpretation of the verb (so called "representation source theme" verbs, cf. Dowty, 1979).

(3) a. John wrote a new book.

b. Sophie has been writing for hours.

(4) a. John built a wooden bookcase.

b. *John has been building for weeks.

(5) a. John built a wall (out of bricks).

b. *John built the bricks into a wall.

(6) a. Mary stacked the blocks into a tower.

b. *Mary stacked the tower.

(7) a. John assembled the sofa.

b. John assembled the individual parts into a complete sofa.

(8) They have already photographed the scene.

Works on scalar change (cf. Beavers, 2008, Levin and Rappaport Hovav, 2010) and dynamic event semantics (Pustejovsky, 2000, Naumann, 
2001) suggest a new understanding of the interplay between verb meaning, event semantics, and argument structure for these predicates, by focusing on the measurement of the change in value over the properties of the participants in each intermediate state during the event. However, they make conflicting statements regarding the attribute available to construct the gradualness or gradience scale for creation verbs, and no general consensus has been reached regarding the role played by arguments in the aspectual profile of the predicate, i.e. whether the scale is lexicalized in the predicate (Hay et al., 1999) or introduced in composition by the object (Rappaport Hovav, 2008, Kennedy, 2009, Levin and Rappaport Hovav, 2010). Finally, their accounts do not provide an explanation of the contrastive data on argument variation introduced in (3) to (8) above.

In the following discussion, we propose a new analysis of these predicates, based on a dynamic interpretation of the event and of the changes the participants undergo while the event unfolds over time, as well as their syntactic realization. We identify the parameters of behavior dictating the expression of change for creation and destruction predicates adequate for this purpose. These parameters are the semantic and syntactic linguistic dimensions as defined by the answers to the following questions:

(9) a. Is the created/destroyed object realized in the syntax?

b. What is the semantic type of the resulting object in a creation?

c. What counts as a result of a destruction act?

d. Does the creation act make use of existing resources, such as material or substance? How are these exploited in the event?

e. Are the resources that are used in the event realized in the syntax?

The answers to these questions will help in creating a coherent classification of change predicates in language. To this end, the major contribution of this study is to characterize each class in terms of its dynamic argument structure (DAS), a representation, the features of which correspond to how the questions in (9) are answered (section 2). We will also show how the information encoded in the dynamic argument structure can be mapped onto a dynamic event representation that tracks the temporal unfolding of the event. The theoretical underpinnings are introduced in section 1.2, while the analysis of members for each of the proposed class is provided in section 1.3 for creation classes and in 1.4 for destruction ones. Section 1.5 examines the consequences of the proposed representation for the computational interpretation of linguistic 
expressions. Section 6 reports our concluding observations.

\subsection{Dynamic Argument Structure}

For the discussion that follows, we develop a model of dynamic selection, our goal being to motivate the typology of change required to describe the linguistic behavior of creation and destruction predicates and their arguments. We will argue that the semantics of change, as expressed in verbal predicates, should include specification of how the event participants behave throughout the event: that is, a participant can stay the same, be modified, come into existence, or be terminated. In the following, we will first review the dynamic model of events with which our model is coupled; then we will outline Dynamic Argument Structure in full and show how is can be mapped onto the structure of the whole event.

\subsubsection{Dynamic Event Structure}

Let us then first examine how change can be semantically encoded in a verbal entry. For the present discussion, we start with the basic framework of event-based verbal semantics, as outlined in Generative Lexicon (henceforth GL, Pustejovsky, 1995), which constitutes the representational framework we adopt in our work. In this framework, event types are defined structurally in terms of a syntax of event and sub-event structure, capturing the conventional Vendlerian Aktionsart-based typology for events.

(10) a. EVENT $\rightarrow$ STATE | PROCESS | TRANSITION

b. STATE: $\rightarrow e$

c. PROCESS: $\rightarrow e_{1} \ldots e_{n}$

d. TRANSITION ${ }_{a c h}: \rightarrow$ STATE STATE

e. TRANSITION ${ }_{a c c}: \rightarrow$ PROCESS STATE

Event structure is but one component of the lexical semantic specification for a predicate $P$, as illustrated in (11), where we see that the complete lexical entry for predicates includes three interrelated levels of specification: argument structure (AS), event structure (ES), and Qualia Structure (QS). 
Dynamic Argument Structure / 5

$$
\left[\begin{array}{l}
P \\
\mathrm{AS}=\left[\begin{array}{l}
\mathrm{A} 1=x \\
\mathrm{~A} 2=y
\end{array}\right] \\
\mathrm{ES}=\left[\begin{array}{l}
\mathrm{E} 1=e_{1} \\
\mathrm{E} 2=e_{2}
\end{array}\right] \\
\mathrm{QUALIA}=\left[\begin{array}{l}
\mathrm{CONST}=\ldots P_{2}\left(e_{2}, y\right) \\
\text { TORMAL }=P^{2} \\
\text { AGENTIC }=\ldots P_{1}\left(e_{1}, x, y\right)
\end{array}\right]
\end{array}\right]
$$

The qualia roles in (11) are temporally ordered, and the predicates associated with each subevent are interpreted as ordered frames of interpretation (Bouillon, 1997). To understand this, consider the proposed representation for the transitive verb kill, denoting a transition, in (12): the AGENTIVE role, introducing the causing act, temporally precedes the FORMAL role referencing the end state (RESTR: < in the ES in (12)) in a complex event structure. In other words, kill denote an event $e$, with a structure of $\left[\begin{array}{ll}e_{1} & e_{2}\end{array}\right]_{e}$, where distinct subpredicates occupy each frame (subevent).

$$
\left[\begin{array}{l}
\text { kill } \\
\mathrm{ES}=\left[\begin{array}{l}
\mathrm{E} 1=e_{1} \\
\mathrm{E} 2=e_{2} \\
\mathrm{RESTR}=<
\end{array}\right] \\
\text { QUALIA }=\left[\begin{array}{l}
\text { FORMAL }=\operatorname{dead}\left(e_{2}, y\right) \\
\text { AGENTIVE }=\operatorname{kill} \_a c t\left(e_{1}, x, y\right)
\end{array}\right]
\end{array}\right]
$$

Without an explicit representation of change of state, however, the representation in (12) does not adequately model change dynamically. For this reason, the concept of opposition structure was introduced in Pustejovsky (2000) as an enrichment to the above representation, making explicit what predicate opposition is lexically encoded in a predicate. For example, the verbs die and kill are both encoded with the opposition structure $[\neg \operatorname{dead}(x), \operatorname{dead}(x)]$, (for their subject and object, respectively), defining the states holding during the sub-events $e_{1}$ and $e_{2}$. The entry for kill restricted to Qualia Structure (QS) in (11) in thus enriched to (13), where e3 is a projection of e1 and part of it.

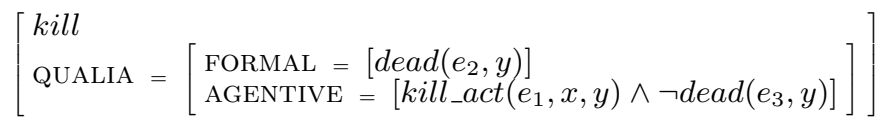

Incremental changes such as climb or fall, however, are still difficult to capture within this representation. In Pustejovsky and Moszkowicz (2011) a first proposal for a dynamically interpreted event structure is presented, which captures the scalar change associated with motion 
events. This is then extended to the class of incremental change predicates in Pustejovsky and Jezek (2011). Pustejovsky (2013) models these insights within a global model of dynamic event structure representation in language.

All these studies accommodate change in the assignment of values to the relevant attributes of the participants in the event being tracked over time. Vendler classes are reinterpreted in terms of dynamic event structures. In a dynamic approach to modeling changes, there is a distinction between formulas, $\phi$, and programs, $\pi$ (cf. Harel et al., 2000). A formula is interpreted as a proposition, evaluated relative to a specific state in the model. Programs are either atomic and complex, where atomic programs are input-output relations interpreted over state-tostate pairings. Within this framework, a state is defined as either a single frame (event) containing a proposition, or an extended sequence of temporally ordered frames containing the same propositional content; this can be evaluated as holding true over multiple adjacent states, which can be handled with an operation of concatenation, + .

The simplest change of state, $\phi$ to $\neg \phi$, is called a simple transition and involves an atomic program, $\alpha$, that changes the content in the first state to its negation in the next state. This corresponds to the basic event structure for achievements, as in (14), where $\phi$ stands the proposition and $\phi$ ? indicates the testing program being performed on the proposition, as described below. Note that in all of the event trees below, temporal sequencing of before_meet is encoded directly in the tree configuration.

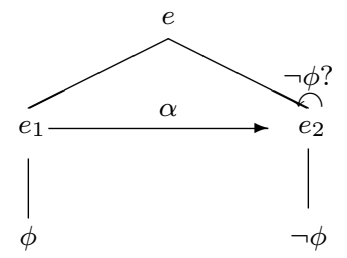

Following Pustejovsky (2013), we call verbs that encode this event type test predicates, because the program they encode is a test that checks whether the change occurring during the execution of the event $\phi ?$ is identical to the distinguished endpoint encoded in the event semantics $\phi$, and stops when this point is reached. In our model, a test program references a nominal scale, a scale that introduces a dichotomy between what did not exist before the event and what exists at the end of it. In our use of the term scale, we depart from the conventional linguistic terminology, according to which a scale is usually understood 
as an ordinal structure (either two-point or multi-point, cf. Rappaport Hovav, 2008), and adopt the distinction between nominal and ordinal scales as discussed in Suppes and Tversky (1989). In this view, a test is a program that constantly checks whether the changes against the goal defined by the nominal scale encoded in the verb is attained.

According to Pustejovsky (2013) we define a process, in dynamic terms, as a sequence of transitions, where at each transition, the value of an attribute $\alpha$ associated with an argument is assigned and reassigned $(\neg \phi$ ?), as there is no terminal test in the proposition $\phi$ expressed by the verb (Pustejovsky and Moszkowicz, 2011) (cf. 15). The incremental change in the attribute associated with the argument is encoded as an assignment program that makes reference to an ordinal scale. Verbs encoding processes are assignment predicates.

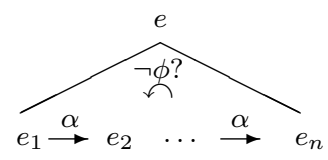

Finally, an accomplishment is an event that makes reference to a preparatory phase consisting of an iteration of changes (ordinal scalar predication), followed by a nominal scalar predication in a resulting state. The iterated assignment stops when the predefined goal or intended state is achieved. This is achieved by testing ( $\phi$ ? in the figure) whether a proposition $\phi$ is true: the process continues while the test is not satisfied $-\neg \phi$ ? - until it finally is. (Pustejovsky and Moszkowicz, 2011). This is represented in (16). Accomplishments verbs encode both an assignment and a test component.

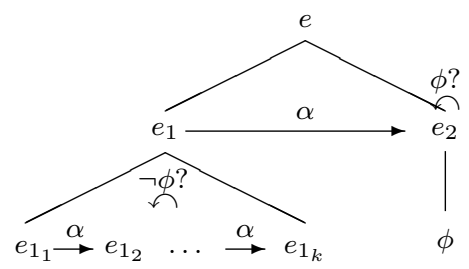




\subsubsection{Dynamic Argument Typing}

As clarified at the very beginning of this section, in this paper we enrich and refine the analysis of change events reviewed in section 1.2.1 above by focusing on the participants of the event. That is, we take into account the argument structure of the verbs in questions, and examine and encode how each participant changes while the event unfolds over time. Particularly, we propose a new representational model for argument structure information encoded in verbs, called Dynamic Argument Structure (DAS). The Dynamic Argument Structure encodes how an argument to an event contributes to the change being expressed. DAS identifies the following properties for each argument to a predicate: the mode of change it undergoes; its semantic role; its semantic type; and whether it is syntactically realized.

We will distinguish four primary modes of change that a participant may assume, each of which refers to the role played by that specific argument as the event unfolds. ${ }^{1}$ They are:

(17) a. Modification

b. Initiation

c. Termination

d. Transfer

An argument undergoes modification (mod) if there is an identifiable attribute whose value is changed during the event. An argument undergoes initiation (init) if it is brought into existence as predicated by the verb, and undergoes termination (term) if the converse is true. An argument undergoes transfer (transfer) when it represents the transfer of information from a source through a medium (both these roles are defined below).

In the representations that follow, we also consider the existence mode. This is not a mode of change: it is a mode that qualifies the resource that exists prior to and independently of the event, corresponding to the available material out of which an object is created (with creation predicates) or to the object which is terminated (with destruction predicates). Notice that there exists an implication between the modes in (17), such that termination presupposes initiation, which presupposes modification which presupposes esistence.

As regards typing, following Pustejovsky (1995) and Asher and Pustejovsky (2006), we assume that the semantic type of an argu-

\footnotetext{
${ }^{1}$ An additional mode, namely Persistence, identifies the property of an argument that is unaffected by the change predicated by the verb throughout the event, such as the Agent in change of state predications. For the current purposes of capturing change in creation and destruction predicates, we can leave this mode aside.
} 
ment is a value selected from an inventory of types in the language. In addition to the Montague types, $e$ and $t$, we use subtypes including complex types (Pustejovsky, 1995), that is, types which are composed of more than one type, such as the type associated with the word book, which include a physical and an informational component. For the present study, the relevant types are summarized in (18):

(18) a. physical object (phys)

b. informational object (info)

c. physical object and informational object $($ phys $\bullet$ info)

e. event (event).

We will also distinguish collections (groups) from individuals, and use entity for the most general type.

Concerning the notion of role, as in most theories where semantic roles are adopted, role assignments are usually unique for each argument to the verb. But as we demonstrate in the discussion below, when events are interpreted dynamically, labels to each argument will reflect the role it plays in that phase of the event, which may change as the event unfolds. We will draw on the following semantic roles for characterizing the behavior of arguments of creation and destruction predicates during an event.

(19) a. Resource: the material or object used to bring about the change of state or result;

b. Result: the outcome of the change of state brought about by the event;

c. Source: Dowty's source of representation (Dowty (1979));

d. Medium: the resource used by an animate object acting intentionally in an event that brings about a new object through the representation of an existing one, for example characters for writing.

There is a clear relation between the roles, particularly between the Resource and the Result; we will illustrate in 1.3 how this is captured formally. In order to keep track of the change occurring in the participants throughout the process, we adopt and extend the notion of dynamic program variable, used in Pustejovsky and Moszkowicz (2011) for motion predicates and in Pustejovsky and Jezek (2011) for general scalar predicates. A dynamic program variable is a variable that keeps track of the current state of what has been "acted on". It may designate either the values of the attribute of the resource being modified - with change of state verbs, or the intermediate stages of the "resultto-be" - with creation or destruction predicates. It is annotated vith a 
vector, $\vec{x}$. For example, in the unfolding process of building a house, the dynamic program variable representing the intermediate stages of the "coming-into-existence house" is encoded as result.

Finally, the feature syn encodes the conditions under which an argument is expressed in the syntax. There are three possible values: expressed, covert, and optional. Both covert and optional arguments are licensed by the predicate and present at the level of semantic representation, but while the former encode participants that can never be expressed, the latter encode participants that may be omitted under specific conditions. An example of covert argument is the dynamic program variable, which is present in the dynamic inspection of the event but not expressed syntactically.

Now let us consider how to encode these features of the arguments to a verb. For this purpose, we use a feature structure representation. The argument-based change profile DAS for a transitive verb, $\operatorname{verb}(x, y)$ is illustrated (20). We use the roles in (19) to label the variables, in order to have a better view of the dynamic behavior of the resource and the result through the change.

$$
\left[\begin{array}{l}
\operatorname{verb}(\mathbf{x}, \mathbf{y}) \\
\operatorname{DAS}=\left[\begin{array}{l}
x: \text { role }=[\text { type, mode, syn } \\
y: \text { role }=[\text { type, mode,syn }
\end{array}\right]
\end{array}\right]
$$

The representation in (20) can be seen as a dynamic way to encode the arguments in the verb's lexical entry, which, as we have seen in (11), also includes ES and QS as representational levels. With respect to the ES, DAS represents an abstraction of the phases of the event as encoded through the arguments. The question then becomes how one integrates the argument-based change profiles into a dynamic event structure representation. We propose that annotating the arguments in the event structure with the modes of change introduced in DAS actually captures dynamics directly, and allows us to recover the dynamic aspects of the event from the annotation on the arguments to the verb.

We introduce and discuss the proposed combined DAS and ES annotation in the following section, where we apply it to the analysis of several subclasses of creation and destruction predicates. ${ }^{2}$

\footnotetext{
${ }^{2}$ For current purposes, in the following we will simplify the representations in $(14),(15)$, and (16), and exclude the notation of the test/assignment components, assuming that they are present according to the model presented in section 1.2.1, namely: simple transitions as in (14) encode a test component, processes as in (15) encode an assignment component and accomplishments as in (16) encode both.
} 


\subsection{Creation Predicates}

In this section we introduce our classification of creation predicates. For each class, we give a definition with examples, then we supply the formal analysis, and the compositional derivations, based the model presented in 1.2.2.

\subsubsection{Creation through Exploitation of Resource}

The verbs associated with this class denote any transformation of an existing object or objects, through: (a) natural growth (generation), (b) aggregation or (c) resource manipulation.

\section{Natural Growth}

There are two aspects of natural growth. One aspect is the natural "manifestation" of an entity out of another by virtue of the character of its inherent properties. Examples of such predicates can be found in Levin (1993) "grow verbs" class, which includes the verbs develop, evolve, grow, hatch, and mature. The other aspect relates to the same process, but focuses on the incremental change that the object undergoes. Both of these aspects are manifested in the two senses of the verb grow. To illustrate, consider the sentences below in (21).

(21) a. A tree is growing in the back yard.

b. A lump grew on the patient's arm.

In (21a), reference is made to the participant which undergoes the modification. This property is reflected in the DAS and the dynamic event structure for the incremental sense of grow, as shown below in (22) and (23).

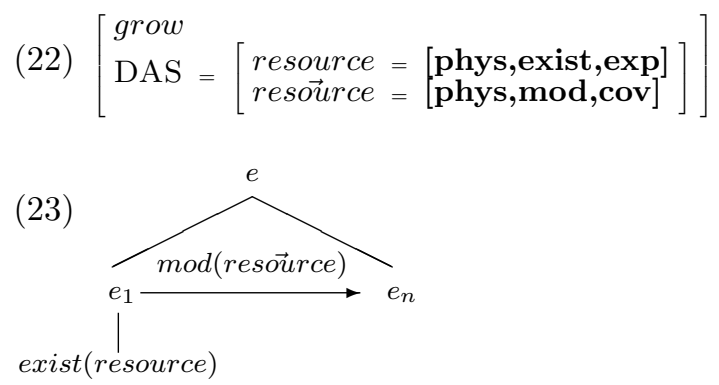

The participant undergoing the change in (21a), i.e. a tree, is interpreted in (22) and (23) as the resource of the grow event. The relation 
between the resource and the dynamic program variable in (22) is captured formally as follows. The resource exists before the event; the modification mode $(\mathrm{mod})$ from the verb's semantics applies to its program variable, i.e. the covert argument that captures the incremental changes the resource goes through as an argument of the grow function. $^{3}$

By contrast, in (21b), an object is brought into existence due to naturally occurring circumstances. The entity (a lump) is initiated through the modification of existing resources, which are left unexpressed. The DAS and the dynamic event structure for the creation sense of grow are represented in (24) and (25) respectively:

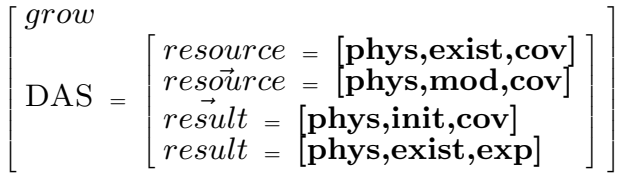

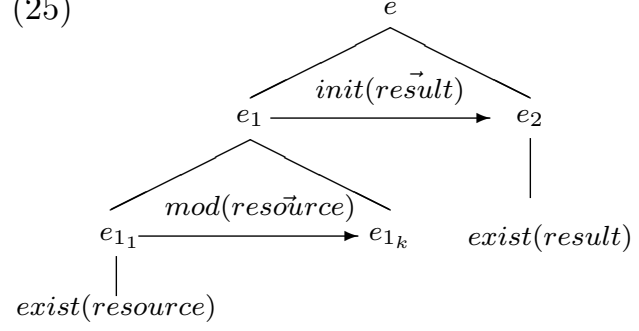

The difference in the two senses of grow, as used in (21), is captured by the distinct DAS representations above. The process reading of grow in (21a) is entailed by the sense of grow expressed in (25), where a new resulting object is identified by virtue of the process. There are two dynamic variables: one encodes the modification of the resource, the other one the coming into being of the resulting created object. The dynamic variables encode the modification of what is changing in the object being tracked, from both the perspective of the initial resource and of the resulting created object.

Compare this to the sentences in (26), where both resource and result objects are syntactically realized.

\footnotetext{
${ }^{3}$ Because of this, we do not annotate the subsequent events following $e_{1}$ with content indicating exist(resource), as it is entailed by the program variable undergoing change through mod.
} 
(26) a. The sapling grew into a beautiful tree.

b. The small boy matured into a healthy man.

c. The storm developed into a hurricane.

For the examples in (26), more so than for those in (21b), we can think of the resulting (created) object that appears in the PP complement as part of the "natural trajectory" of the resource object: that is, the characterization of this individual as a sapling is more accurately replaced by the sortal characterization brought about by tree. ${ }^{4}$ The representation of the DAS of the expression in (27) is given in (28).

(27) The sapling grew into a tree.

$$
\left[\mathrm{DAS}=\left[\begin{array}{l}
\text { resource }=[\text { phys, exist,exp] } \\
\text { resource }=\left[\begin{array}{l}
\text { phys, mod,cov] } \\
\text { result }=[\text { phys,init,cov }] \\
\text { result }=[\text { phys, exist,exp] }
\end{array}\right]
\end{array}\right]\right.
$$

In (28) the sapling (as subject) is the syntactic manifestation of the resource. The dynamic variable resource keeps track of the accumulated changes in value of the resource, as viewed from the predication of the result, i.e., the initiation of the tree $($ result $)$. While the resource continues on in the guise of resource, reference to the nominal classification of it is no longer possible as soon as the new type, i.e. result, is initiated. The corresponding dynamic event structure mapping is the same as that in (25).

The lexical typing for this class of verbs will have to reflect the polyadicity demonstrated by both pure intransitive and PP-selecting intransitive forms. This can be accomplished by assuming that in the case of pure intransitives as in (21b), the last subevent is headed as a result of pragmatic factors, resulting in the realization of the corresponding result argument, while PP-selecting intransitive forms as in (26) the whole event is headed, resulting in the realization of both the resource and the result. We borrow the notion of event headedness from Pustejovsky (1995), 69-73; it is the property of a subevent to act as the most prominent part of the matrix event from a structural point of view, contributing to the focus of the interpretation and determining the realization of the arguments associated to it. It is marked with $\mathrm{e}^{*}$. We propose here that headedness is not only assigned lexically but can also be assigned compositionally and pragmatically. Taking headedness into account, the representation of (27) is presented below, where * marks the head subevent.

\footnotetext{
${ }^{4}$ The natural stages in a life cycle would be conceptualized as a large semantic structure. A possible model is the projective structure from Pustejovsky (1995).
} 


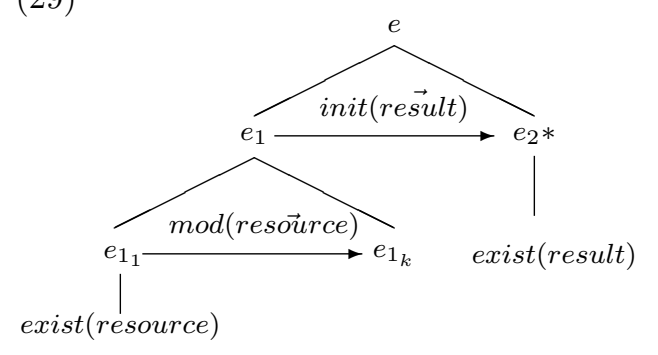

\section{Aggregation}

This class refers to the assembly or coming together of existing resources to create a distinct object, identified by a newly introduced expression. Included in this class are the verbs assemble, collect, gather, and congregate. The resource encodes the referential objects available prior to the change, and can be either presupposed or directly selected as an argument. For those verbs that have intransitive forms (assemble and gather), this distinction can be clearly illustrated with the examples in (30).

(30) a. A crowd assembled / gathered in the lobby.

b. The students assembled / gathered in the lobby.

Notice that in (30a), the subject directly denotes the object formed by the event, i.e. the result. That is, the crowd is brought into existence by the assembling or gathering events. For this verb class, the key feature is, of course, the relationship between a set of individuals serving as the resource type, and a group denoting the result type. Even though the selectional restrictions on $(30 \mathrm{~b})$ reflect the former constraint, it is possible to interpret the NP in subject position (the students) as being coerced into a group interpretation by the verb, i.e., we assume that assemble in its intransitive use types its subject as group. This would then be reflected in the DAS representations below, where we encode a distinction between the typing restriction of $[+$ plural $]$ from the predicate assemble onto its resource, and the output condition (resulting type) from the computation, viz., a group or crowd. Consider first (31) and the argument change profile in (32) (where ind $[+\mathrm{pl}]$ stands for a plurality of individuals).

(31) A crowd assembled in the lobby. 
Dynamic Argument Structure / 15

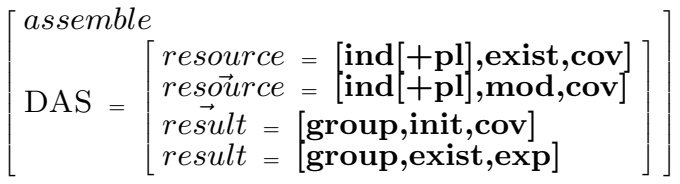

The corresponding Dynamic Event Structure is in (33), where the headed subevent is $e_{2}$, i.e., the one associated with the result argument:

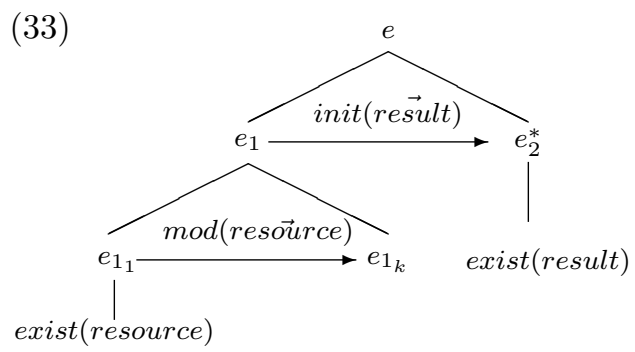

Now consider the sentence in (34), where the result is left covert, but the resource is expressed syntactically.

(34) The students assembled in the lobby.

The DAS of (34) is the same as (32), with the difference that the resource is expressed instead of the result:

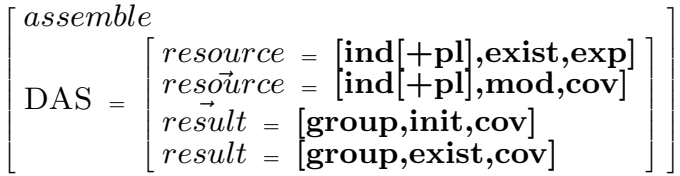

The dynamic event structure is also the same as (32), with the exception that the head of the event is in this case $e_{1}$, i.e., the subevent associated with the resource argument. ${ }^{5}$

\footnotetext{
${ }^{5}$ Notice that transitive forms for such verbs allow alternations on the direct object, as illustrated by the sentences in (36), where in (36a) the direct object encodes the result, whereas in (36b), it encodes the resource. Both the resource and the result are instead expressed in (36c):

(36) a. Mary assembled the bookshelf.

b. Mary assembled the parts into a bookshelf.

c. Mary assembled the bookshelf out of old pieces of wood.
} 


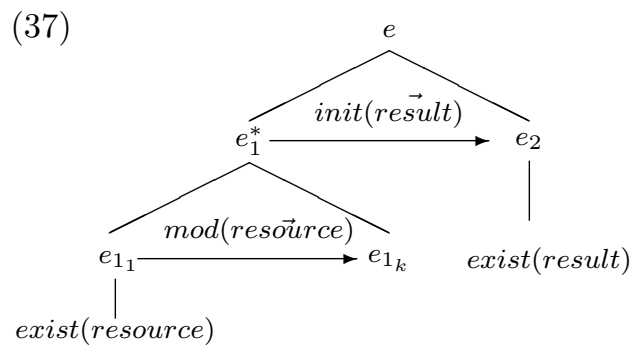

An alternative account to the coercion analysis we propose is to assume that the event structure of assemble is underspecified lexically, and allows for two distinct syntactic realizations, depending on which subevent is under focus and acts as head. This analysis could be preferable to account for cases in which both the resource and the result are expressed, as in "bits and pieces waiting to be assembled into a whole", in which the whole event would be analyzed as headed. However, corpus analysis performed on the EnTenTen corpus queried through the Sketch Engine tool (Kilgarriff et al., 2014) has shown that assemble has a clear tendency to select collecting nouns as subjects, such as congress, parliament, crew, congregation, army, fleet, team, and others, leading us towards the coercion account.

\section{Resource manipulation}

We turn now to the major group within the class, namely resource manipulation. This includes what are conventionally viewed as creation predicates in the literature (Dowty, 1979, Jackendoff, 1990, Levin, 1993, von Stechow, 2001, Piñón, 2008), with verbs such as build, knit, bake, create, produce, compose, and so on (cf. the "create" verbs in Levin, 1993). Obviously, this class is not a homogeneous one and there are many semantic and syntactic distinctions to be made within it (for an overview, see Bisetto and Melloni, 2007, Jezek, 2014). In this section, we examine how the model developed here can characterize the common elements of the verbs within this class, as well as how they differ amongst themselves. In particular, the major distinctions within this class that we wish to characterize formally are the difference in behavior in object selection/dropping, and the alternation between a creation sense and a change of state sense, as in bake.

Traditional analyses of these verbs view them as differing from regular accomplishments in that they take a participant that measures out the event, i.e., an incremental theme argument (Dowty (1991)). Recent work on scalar change offers a new way to look at the inherent temporal 
properties of these verbs, none of these analyses, however, can account for the long recognized distinction (Dowty, 1979, Resnik, 1996) between transitive creation verbs that frequently exhibit a so-called object-drop alternation (e.g., draw, write, knit, paint) and transitive creation verbs that typically do not (e.g., build, construct, create, make, produce). This distinction is illustrated below, where build and construct are contrasted with knit (see also Section 1).

(38) a. John built a wooden bookcase.

b. ${ }^{*}$ John has been building for weeks.

(39) a. The city constructed a new fence around the reservoir.

b. *The city constructed in the spring.

(40) a. Mary knitted a sweater.

b. Mary knitted yesterday evening. ${ }^{6}$

Consider first knit in the context below:

(41) Mary knitted yesterday evening.

In our model, predicates like knit Lexically denote a process, i.e. they are assignment predicates which leave a trail of the process, namely the object being knit. This argument is unexpressed in the syntax but present in the inspection of any state of the process. As referenced above, this argument is encoded in the DAS in (42) as a dynamic program variable, (resource), next to the resource argument available at the beginning of the event. ${ }^{7}$

$$
\left[\begin{array}{l}
\text { knit } \\
\text { DAS }=\left[\begin{array}{l}
\text { resource }=\left[\begin{array}{l}
\text { phys, exist, cov } \\
\text { resource }
\end{array}\right] \\
\text { phys, mod,cov }
\end{array}\right]
\end{array}\right]
$$

The corresponding dynamic event structure is in (43):

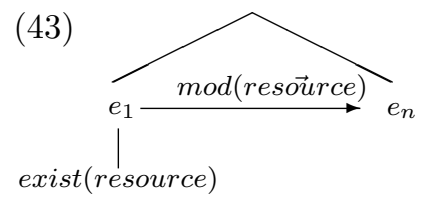

\footnotetext{
${ }^{6}$ Note that it is not totally impossible to make up a context in which build and construct are constructed without direct object, but differently from knit, the referent of the argument must in this case be retrieved from the immediate situational or discourse context, it is in other words a pragmatic deletion; moreover, such contexts would definitely be exceptions and not the norm, as disclosed by corpus evidence.

${ }^{7}$ Recall from section 1.2.2 that in this contribution, we factor out the persistent Agent and focus on the Resource and the Result arguments, i.e. the participants undergoing change.
} 
The representation in (43) resembles the one proposed for incremental grow in (23), the difference being that with grow, the resource is realized in the syntax as subject.

Now consider build. The chief characteristic of predicates such as build is the explicit encoding of a test, in addition to the assignment component, in the verb's semantics as their selected direct object argument. It is typed as a quantized physical object. This distinguishes these predicates from assignment predicates such as knitWe claim that it is this reference to a test that makes object-drop typically impossible for such predicates.

Thus, a verb such as build leaves a trail, generated by the assignment component associated with the process subevent, while testing the value of this trail against the semantics of the distinguished value denoted by the direct object. Therefore, it has an incrementally created (trail) argument, denoted by a program variable, as well as the argument identified with the test.

The DAS for this verb given in (44) introduces different modes for distinct arguments: first, an initiation mode will characterize the creation of the argument, expressed as the direct object; the resource is annotated as existing independently of the event, but modified via the program variable (resource) that will become the resulting creation (result).

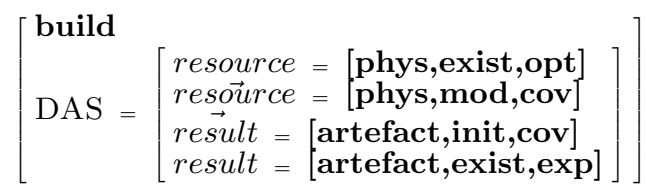

Notice that build allows for the optional expression of the resource argument. This can be accomplished in one of two ways, illustrated in (45) below.

(45) a. John built a wooden bookcase.

b. John built a bookcase out of wood.

Let us now look how the DAS of build maps onto the dynamic event structure. We assume that build is lexically an accomplishment (head on e2); during the process component, the resource is modified, until the new object is initiated. 
Dynamic Argument Structure / 19

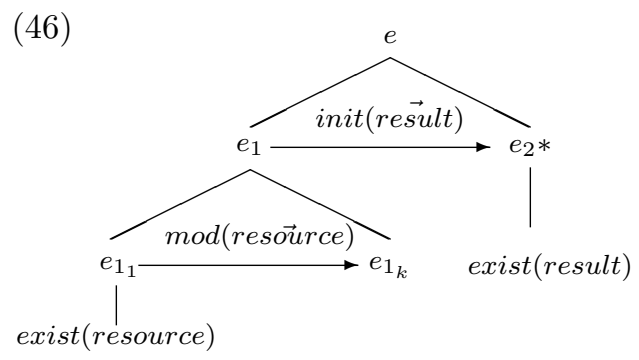

Now that we have introduced a formal distinction between assignment predicates such as knit and test predicates such as build, it is necessary to explain why verbs in the former class can typically take NP direct objects and, in case these are quantized, acquire an accomplishment reading, as shown in (47).

(47) Mary knitted a sweater last night.

To answer this question, we argue that there are two semantic interpretations to constructional transitivity in creation predicates.

(48) a. Selection of a test (as a quantized NP) as an argument to a transition predicate;

b. Expression of a test as the quantification resulting from an assignment predicate.

The first interpretation is employed by direct argument selection in transitive creation predicates, e.g., build. The second is what we see at play in (47) above, and for this interpretation, there are at least two distinct grammatical strategies, listed below, that introduce a test over the activity denoted by the matrix predicate.

(49) a. ARGUMENT INTRODUCTION; this creates a test by making reference to a nominal scale denoted by the NP in object position; knit a sweater.

b. ADJUNCT INTRODUCTION; this creates a test with a measure phrase relevant to the trail left by the assignment predicate, namely the object being knit; knit until noon. 
Consider again the accomplishment interpretation available with the verb knit in (47) in the light of what we just said. In the example in (47), we see the composition of the process reading of knit with its argument, resulting in an interpretation as an accomplishment, where the result argument, the sweater, is brought into existence, i.e., initiated. The DAS for the expression is as follow:

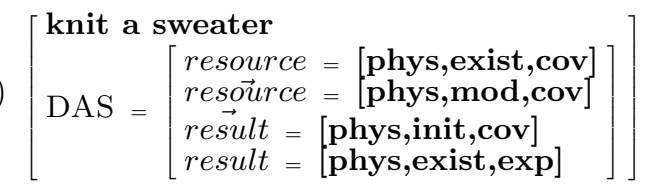

The corresponding dynamic event structure of the compositional expression knit a sweater is the same as that for build, as shown in (46). In other words, with predicates such as knit, argument introduction creates a test which determines when the incremental directed process should terminate. This shifts the scale of interpretation for these predicates from ordinal to nominal, something referred to as scale shifting in Pustejovsky and Jezek (2011).

To conclude this section, we turn to the issue of how we can model verbs which exhibit an alternation between a change of state sense and a creation sense, namely, the polysemy exhibited by bake in (51a-b) and (51c) (cf. Atkins et al., 1988):

(51) a. Mary baked the potato.

b. Mary baked a cake.

First, we must adequately model how the object in the change-of-state sense of bake in (51a) is being modified. We will treat the resource in the event as undergoing change, through a modification function. As with build, we model the modification function as being associated with the covert program variable (resource) introduced by the process. We can state the DAS and the dynamic event structure for the process reading of bake with object as follows, where the resource is expressed as direct object:

$$
\left[\begin{array}{l}
\text { bake the potato } \\
\text { DAS }=\left[\begin{array}{l}
\text { resource } \\
\text { resource }=\left[\begin{array}{l}
\text { phys, } \mathbf{\text { exist }}, \mathbf{e x p} \\
\text { phys }, \text { mod }, \mathbf{c o v}]
\end{array}\right]
\end{array}\right]
\end{array}\right]
$$


Dynamic Argument Structure / 21

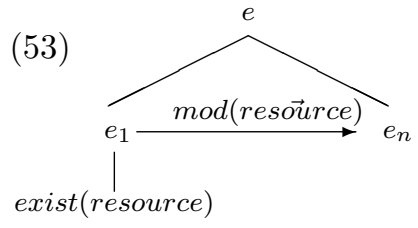

Under the create interpretation for bake (51b), the resource (the ingredients being used) is modified but not expressed, while the output type is initiated, from the modified resource. The DAS for the create sense of bake reflects this observation. The accomplishment reading of $(51 \mathrm{~b})$, is shown below. The creation sense is attained in co-composition through introduction of an argument typed as food artifact, such as bread, cake, cookies, etc. Following Pustejovsky (1995), we use co-composition to indicate the compositional operation where multiple elements behave as functors; in this case, the argument acts functionally to co-determine the meaning of the verb in the context of use.

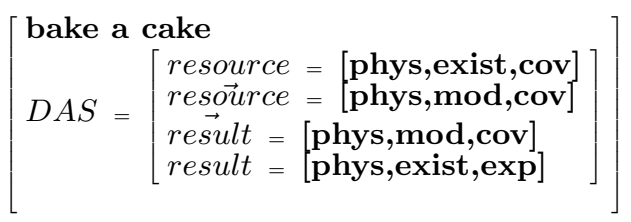

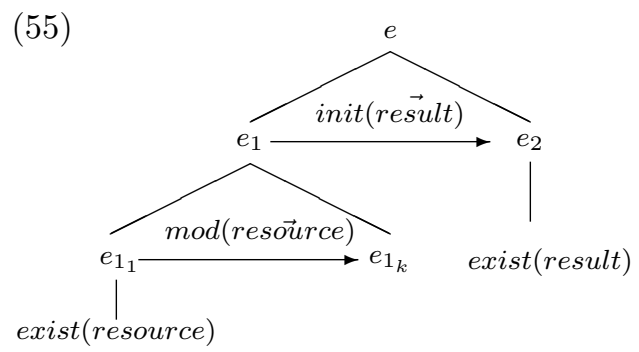

\subsubsection{Creation through Representation}

In this section, we discuss the class of predicates that bring about representational artifacts. This class of predicates involves the act of creating a representation of a source object, either real or imagined. ${ }^{8}$ In the model proposed here, the creation of a representation of an object

\footnotetext{
${ }^{8}$ See Dowty (1979) and Dowty (1991) for early work on the interaction of "Representation" and "Source" in such verbs.
} 
acting as source involves the transfer to information from the source to the resulting created object through a medium.

For our present discussion, we will distinguish three classes of representational creation predicates, mostly based on their behavior at the syntax-semantics interface, i.e. whether their direct object expresses the representational created artifact (56a), the source of the representation (56b) or can alternate between the two (56c):

(56) a. RESULT VERBS: write.

b. SOURCE VERBS: photograph.

c. ALTERNATING VERBS: draw, paint, film.

We first consider the subset of class (56a) involving incremental themes of representation (cf. Dowty, 1991), namely verbs such as write. This class is very similar to the object incremental theme verbs studied above, such as knit, except for two parameters: (a) the source is not necessarily a physical object and (b) the created object is a representational artifact. Such verbs typically exhibit also a variant with no expressed created object, as repeated below.

(57) a. Mary wrote all morning.

b. Mary wrote a letter last night.

When we talk about write, we assume that we are utilizing a script (syllabary, characters, alphabet, semaphores; a medium in our terms) that expresses the interpreted signs in a presupposed language. The act of writing is to create the physical representational manifestation of the information that was present or being thought up (mentally), and that now is "made manifest" on the paper. The basic DAS for (57a) is shown in (58): the source is optional (in the sense that it cannot be expressed as a direct object but it can surface as a prepositional object: see "write about her mother") as well as the medium (see "write on paper"); the result program variable tracks the creation of the complex object derived by representing the source through a medium.

$$
\left[\begin{array}{l}
\text { write } \\
D A S=\left[\begin{array}{l}
\text { source }=[\text { info, exist }, \text { opt }] \\
\text { medium }=[\text { phys, exist }, \text { opt }] \\
\text { resource }=[\text { physoinfo,transfer,cov }]
\end{array}\right]
\end{array}\right]
$$

The corresponding dynamic event structure representation is as follows: 
Dynamic Argument Structure / 23

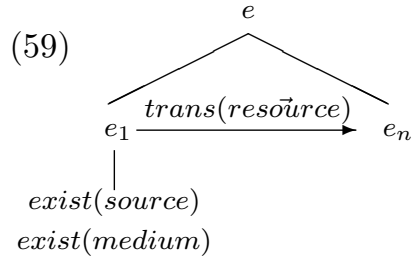

Also the compositional behavior associated with object selection for this class is similar to what we saw for knit, namely, object selection as seen in (60) is the compositional introduction of a test through a quantized direct object.

(60) Mary wrote a letter last night.

The DAS for the variant with introduced object of this verb type is shown below.

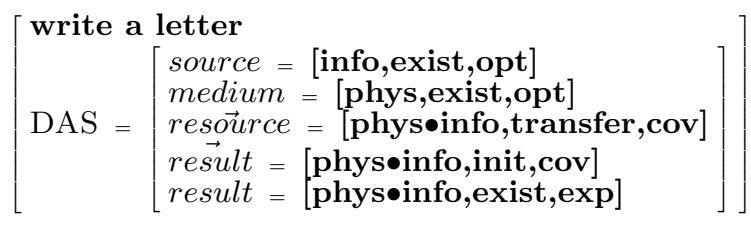

The dynamic event structure representation is as follows. Note that the head of the dynamic event structure is $e_{2}$, i.e. the subevent associated with the argument which is realized as direct object.

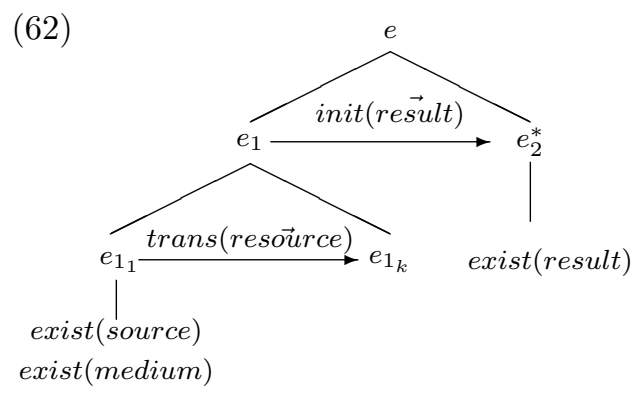

Consider now the second type of representational creation predicate from (56b) above, namely, the representation source verbs. In this class, the output object, i.e., the representational artifact cannot be expressed syntactically. This is a hallmark of representation source predicates, where the syntactic focus is on the source of information rather than the resulting object. Consider the following examples illustrating this well-known property. 
(63) John photographed Mary.

This subclass can be defined by the medium on which the information is captured. The medium is an argument that cannot be expressed because it is already incorporated in the root of the verb, i.e. it is a covert argument in our terminology.

The DAS of photograph can be given as follows. Note that the source ("Mary") is expressed, while the medium and the result remain covert, as well as the program variables. ${ }^{9}$

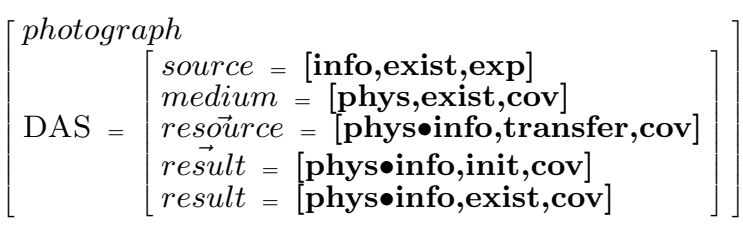

The event representation is given below. Note that in this case the heading event is $e_{1}$, i.e., the subevent associated with the source, which is realized as direct object.

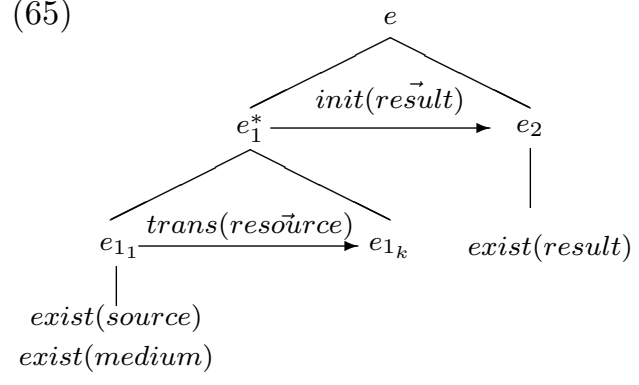

A third class is represented by verbs which permit reference to either the source or to the result of the creative act in direct object position, i.e. they alternate between a variant with the source as direct object, and a variant where the direct object realizes the created representational artifact, i.e. the result. An example is in (66) for paint, where the expressed argument in direct object position is the source: ${ }^{10}$

\footnotetext{
${ }^{9}$ The type of the source can be either a physical object or a situation or event. We will use info as a cover term to indicate the information about the object or the event that is being reproduced. The important thing to realize here is that the source provides the information for the resulting representational artifact, the phys info that is created by the photographing event.

${ }^{10}$ Note that as with write, paint is frequently found with no object, suggesting that it lexically encodes a process (see "He painted all morning"). Differently from
} 
(66) John painted the mountain he could see from the window.

The DAS for paint in (66) is illustrated below, where the source is typed info, i.e. information about the interpreted object or event, and the result is syntactically covert, i.e. not expressed.
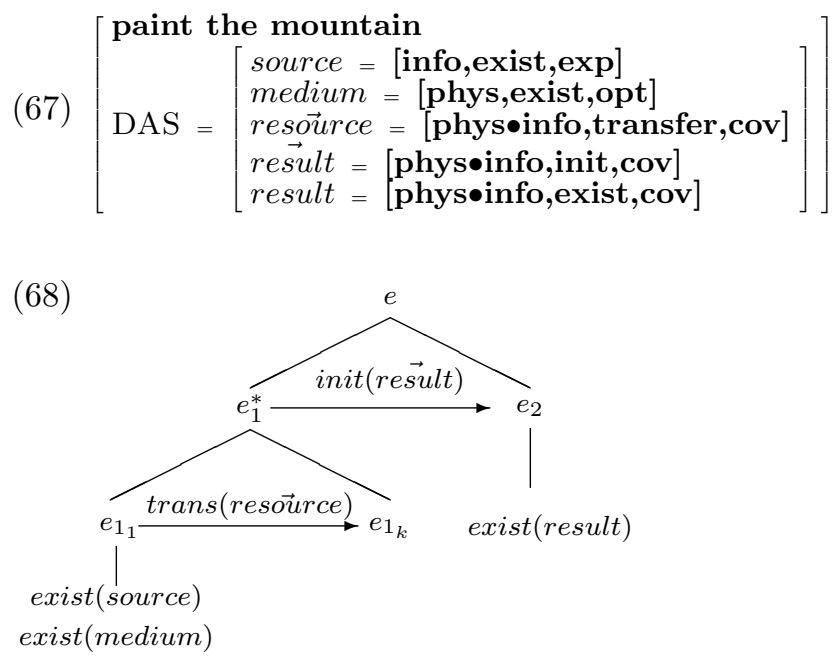

In the case of alternating verbs, it is at the level of event structure that argument realization is defined, i.e. the head of the event will be on $e_{1}$ in the case of the representation source variant (as in (66)), and on $e_{2}$ in the case of the representation result variant, as in (69) below.

(69) Mary painted a portrait. ${ }^{11}$

Another verb within the class is film, as in (72):

(72) a. Mary filmed the wedding. (source)

b. Mary filmed a movie. (result)

write, however, paint allows the direct object to express either the source or the result, when realized, whereas write only allows the result as direct object.

${ }^{11}$ Notice that when the object position is occupied by the resulting representational artifact, the source may still be expressed as an adjunct or a complex NP, as in (70):

(70) Mary painted a portrait of a woman.

Finally, notice that the verb paint also allows reference to the medium being used in the activity, regardless of which argument is expressed in direct object position, i.e., the source or result.

(71) a. John painted the mountain in oil.

b. Mary painted a portrait in oil. 
Because in (72b), the initiated representation-artifact (the movie) is expressed, the DAS is distinct from that of photograph in (64) and is parallel to that of write in (61). ${ }^{12}$

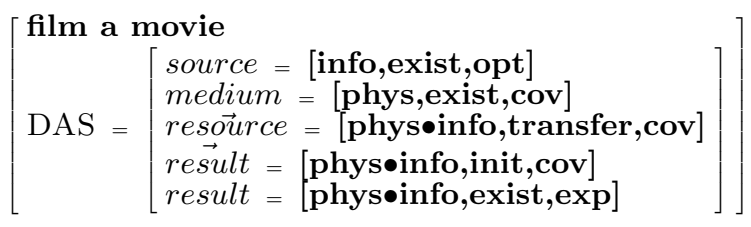

Moverover, in the dynamic event structure $e_{2}$ is the head instead of $e_{1}$ in (68):

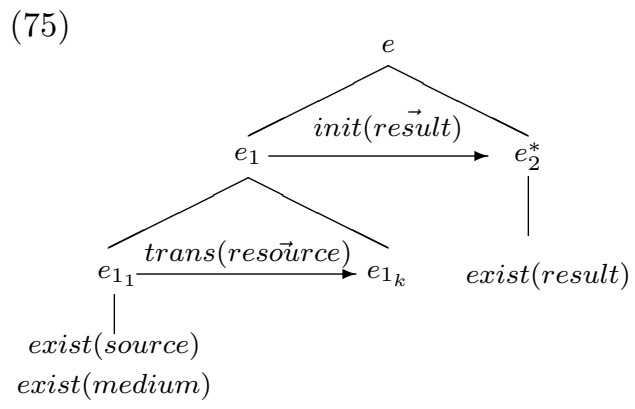

Finally, the verb copy stands out as a special case. The difference between copy and the rest of the class of representation verbs, is that the former presupposes the existence of an object that is already typed as physøinfo, where phys can be media of different kinds. In the case of copy, the medium is not specified in the argument structure of the verb as a separate participant, as in the case of photograph, tape, and record. What copy reports is the pure re-creation of an object, by way of representation. Consider (76):

(76) Mary copied the file.

The corresponding DAS is:

\footnotetext{
${ }^{12}$ Notice that the source may be expressed as an adjunct or a complex NP, as in (72):
}

(73) Mary filmed a movie of the wedding. 
Dynamic Argument Structure / 27

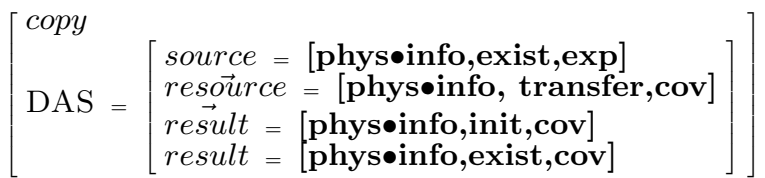

The dynamic event structure representation is as follows:

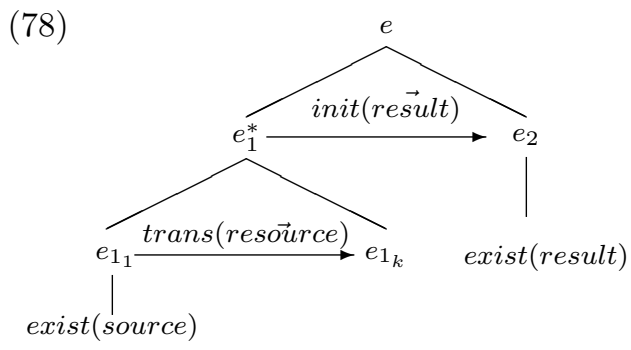

\subsection{Destruction Predicates}

In this section, we illustrate briefly how the dynamic argument structure and the associated typing assignment can be employed to differentiate the various destruction predicates. The purpose of this discussion is to demonstrate the application of the typing strategy developed in the previous sections to destruction predicates generally.

Traditionally, the destruction of an object is seen as the act which takes that object out of existence (terminates it, in our terms). In our model, we examine destruction acts in more detail, and distinguish different kinds of destruction predicates depending on the type of result they encode. We claim that destruction predicates may either focus on what is being done to the pre-existing object (the object being destroyed, identified as the input/resource variable in our model), or focus also on what is being brought about by the activity (what is the output/result of the termination of the input object). In this perspective, as observed in Jackendoff (1990), p.118, destruction predicates are also creation predicates, since the process of transformation can be viewed as creating a result, although these verbs cannot express a created "product" (see also Levin, 1993). The issue is, of course, one of whether the language makes any explicit or implicit reference to the new object resulting from the destruction of the old, if there is one.

Another point we focus on in our classification is whether the predicate focuses on the physical integrity of the object undergoing the change (i.e. its Formal quale, according to Pustejovsky's qualia the- 
ory), or primarily on its ability to be used for its purpose (i.e. its Telic quale) while still referring to modification over the formal aspect.

\subsubsection{Destruction through Modification}

This class will include any process resulting in the termination of an object being classified as a given sortal type, through: (a) dis-aggregation; or (b) termination.

\section{Dis-aggregation}

This is the disassembly of an existing object, into its component parts. There are two grammaticalizations associated with verbs such as disperse, just as there are with assemble, its inverse. The object being transformed and terminated can be selected (79a), or its component parts can be coerced (79b).

(79) a. The crowd dispersed suddenly.

b. The students dispersed.

The DAS for the sense in (79a) is shown in (80) below: ${ }^{13}$

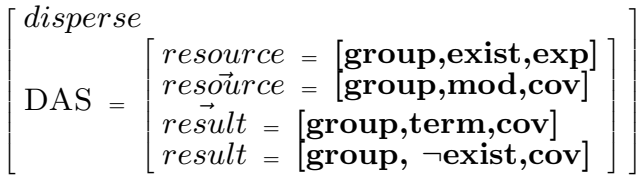

The event structure representation is:

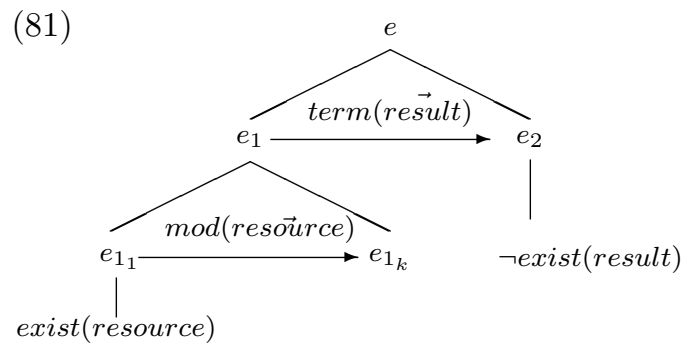

It should be pointed out that, while the original group interpretation for the resource argument in (80a), the crowd, is terminated by the re-

\footnotetext{
${ }^{13}$ If we assume that opt is the value for syntactic expressibility (see section 1.2.2), giving rise to cov or exp, then we must mention that the subject in English must be expressed, and this is an independent principle, not expressed in the lexical entry for a verb such as disperse. What is expressed here is the underspecified nature of whether the subject is a resource or a result role.
} 
sult of the event of dispersing, the component parts (i.e., the individual members of the crowd) will still exist.

\section{Termination}

These verbs report events in which an entity is taken out of existence. Characteristic verbs in this class include the predicates destroy, demolish, topple, and dissolve, as shown below.

(82) a. The earthquake destroyed Mary's house.

b. The fire demolished the church.

c. The winds toppled the tower.

d. Mary dissolved the tablet in water.

With the predicates in (82), the expression of the resulting material (i.e., the output object) is usually odd (83), and remains hidden (Jackendoff 1990, 118).

(83) *The earthquake destroyed Mary's house into pieces.

The syntactic focus is on the resource, while the semantic focus is on the result state of the transformation of this resource: i.e., non-existence, which acts as a test to the expression. When this result state is reached, that is, when the object is no longer classifiable as the nominal it was typed as the resource, the test is passed. The object is now any number of things, should there exist nominal classes to describe it, but the predicate identifies it by what it no longer is.

The DAS representation is as follows:

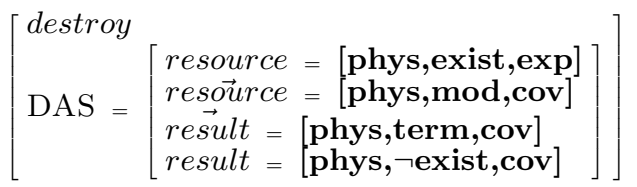

The dynamic event structure for destroy is the same as that given for disperse in (81) above. As with build, verbs such as destroy denote a directed process which is measured against a defined test, introduced explicitly by the state obtained in the absence of the direct object argument.

Consider now inchoative forms of the verb break. There appear to be at least two senses of break: (a) the physical integrity of the object is changed substantially, as in (85a) below; and (b) the ability to use the object for its purpose is no longer possible, as in (85b). ${ }^{14}$

\footnotetext{
${ }^{14}$ There also seems to be cases where both entailments are simultaneously present, as in "Susan broke her glasses". We will discuss them here separately, although we are aware that they may add up.
} 
(85) a. The tree broke in two.

b. My computer broke back in July.

Either sense can be considered a destruction predicate, but with different consequences. For the present discussion, we will focus on the sense in (85b), represented in (86), where result $_{Q_{T}}$ stands for the Telic Quale of the result, which terminates as a result of the event:

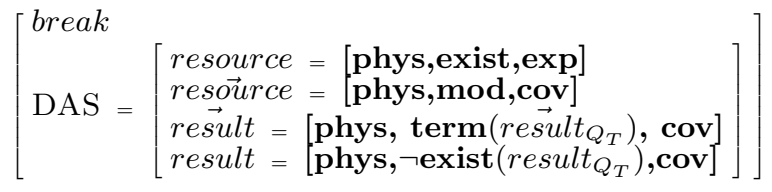

In (87) we report the proposed representation for the dynamic event structure of (85b), enriched with the modes of the arguments:

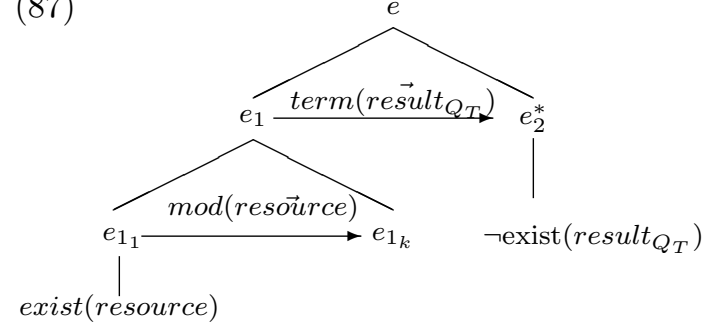

Consider now the following example:

(88) The jar crashed on the floor and broke into fragments. ${ }^{15}$

While destroy verbs do not allow the expression of the material entity resulting from the change as an optional prepositional phrase (see example (83)), break verbs (see Dixon, 1991, 119) appear to allow it.

The representation of the DAS and the event structure of the expression in (88) are (89) and (90), where result $Q_{C}$ encodes the Constitutive Quale of the result, which makes reference to the pieces resulting from event, namely the fragments in (88):

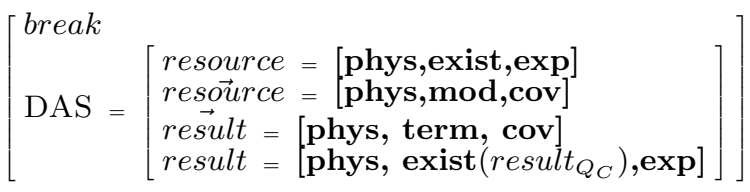

\footnotetext{
${ }^{15}$ Note that when the resulting parts are expressed, as in the example above, break may also acquire the meaning of 'divide', as in "Mary broke the chocolate into small pieces"; we will not discuss these intentional uses of break here.
} 
Dynamic Argument Structure / 31

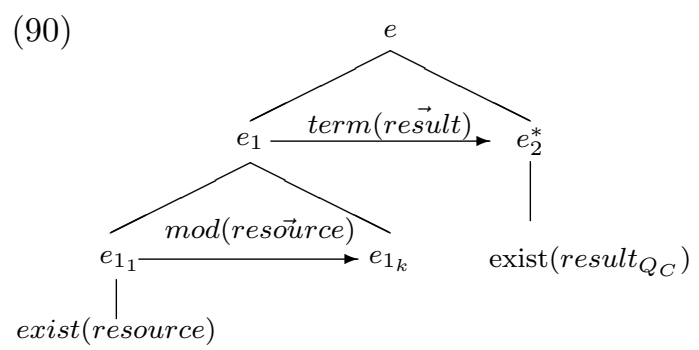

\subsection{Computing with Dynamic Argument Structure}

In the discussion above, we have focused on the role that dynamic argument structure plays in mediating the mapping of a predicate's arguments to syntax. There are, however, some interesting consequences of this representation to the computational interpretation of linguistic expressions. In particular, the enriched argument structure encoding has relevance to the problems of textual entailment and inference (Dagan et al., 2006). "Textual entailment" has come to be associated with the task of performing a range of inferences (e.g., entailments, presuppositions, implicatures) associated with the interpretation of natural language text. For example, in the sentence pairs below, a text (T) is asserted and an hypothesis $(\mathrm{H})$ is proposed, for which the veridicity must be determined.

(91) a. T: The allies destroyed the planes during the bombing.

b. $H$ : The planes took off for raids.

c. $T \not \models H$

(92) a. T: The drought killed the trees.

b. $H$ : The trees are dead.

c. $T \vDash H$

(93) a. T: Mary took apart the computer to repair it.

b. $H$ : The computer is working.

c. $T \not \models H$

For those verbs represented by the classes of change studied in this article, the inferential tasks associated with text entailment above are facilitated by the labeling of change profile features associated with resource and result arguments. For example, in both (91c) and (93c), the predicates are typed as Termination and Dis-aggregation predicates, respectively. The consequence in each case is a lack of reference for the subject in both hypotheses. For (92c), the entailment of non-existence 
is consistent with the hypothesis presented. The dynamic argument type for the argument undergoing change is illustrated below.

(94) NP $=$ [RESOURCE: exist $\mapsto$ RESULT: $\neg$ exist $]$

Briefly, another phenomenon that can benefit from a systematic DAS encoding in the verb involves tracking the predicative status of an argument undergoing change in a discourse. Consider the coreference distinctions illustrated in (95) and (96).

(95) The audience ${ }_{i}$ applauded to show $\operatorname{its}_{i} /$ their $_{i}$ approval.

(96) a. The audience ${ }_{i}$ left the music hall.

b. ${ }^{*} \mathrm{It}_{i}$ then went home.

c. They ${ }_{i}$ then went home.

In Pustejovsky (2000), the persistence of the entity audience has been "gated" (or terminated, in the present model) by the verb in (96), hence preventing coreference in (96b). The dynamic argument structure for this predicate represents this by dis-aggregating the entity, and rendering it unavailable for coreference.

Finally, one further application of dynamic argument structure involves the identification of semantic roles for the participants of an event, known as Semantic Role Labeling (SRL). SRL is a computational task that involves identifying a verb's participant roles and labeling them correctly, regardless of their syntactic realization in a sentence. To illustrate the basic SRL task, in the sentence pairs below, not only is it important to recover the correct labeling of an argument's semantic function relative to the verb, but this must be performed in diverse syntactic contexts.

(97) a. The burglar [AGENT] broke the window [THEME]. b. The window [THEME] broke suddenly.

Adopting the DAS strategy outlined above affords us the opportunity to encode dynamic aspects of how the argument changes over the course of the event within the context of a semantic role labeling exercise. For example, consider the aggregation-class verb, assemble, discussed in Section 1.3 above. From the DAS for this verb, we could (simplifying a bit), explicitly encode the argument as undergoing a specific change, directly in the SRL label, as illustrated in (98) below.

(98) A crowd [RESOURCE: ᄀexist $\mapsto$ RESULT: exist] assembled in the lobby.

Similarly, for the sentence above in (97a), the change of state of the THEME is encoded through a dynamic argument structure value: 
(99) a. The burglar [AGENT] broke the window [RESOURCE: exist $\mapsto$ RESULT: ᄀexist].

Such a direct encoding of the dynamics of the event would also allow for a richer subevent interpretation from lexical resources, such as VerbNet (Pustejovsky et al., 2016), a topic we are presently exploring.

\subsection{Conclusions and future work}

Our purpose in this paper was to provide a new representation of arguments for verbs, in which the changes that the participants undergo during the event are encoded directly in the argument structure. To this end, we have presented a classification of verbs of change and modification, that focuses on a dynamic interpretation of the change induced by the predicate on its arguments. We have looked at the major classes of creation and destruction predicates, in terms of a dynamic argument structure, i.e. a change profile of the arguments, which tracks how the changed objects behave dynamically throughout the event. We have mapped this representation onto the event structure representation proposed in Pustejovsky and Moszkowicz (2011), which includes a scalar component conceived in terms of assignment and testing programs. In this way, we have enriched this latter representation with the modes of change, and integrated it in the DAS structure. Moreover, we have refined the proposal that different kinds of scale can be referenced in a compositional process, and that the compositional shifts in the scale of interpretation can be analyzed as scale-shiftings, as put forth in Pustejovsky and Jezek (2011). Finally, we have shown that the proposed representation has potentially interesting consequences for the computational interpretation of linguistic expressions, and in particular we provided an example of an application focusing on how the NLP tasks of textual entailment and semantic role labeling could exploit richer argument structure information.

\section{Acknowledgments}

We would like to thank the participants of the "Scalarity in VerbBased Constructions" Workshop, held at Heinrich-Heine-Universität Düsseldorf, April 7-8, 2011, where a preliminary version of this work was presented. We would also like to acknowledge the fruitful comments of two anonymous reviewers. All remaining errors are, of course, our own.

\section{References}

Asher, Nicholas and J. Pustejovsky. 2006. A type composition logic for gen- 
erative lexicon. Journal of Cognitive Science 6:1-38.

Atkins, Beryl T, Judy Kegl, and Beth Levin. 1988. Anatomy of a verb entry: From linguistic theory to lexicographic practise. International Journal of Lexicography 1(2):84-126.

Beavers, John. 2008. Scalar complexity and the structure of events. In T. H.Z. Johannes Dölling and M. Schäfer, eds., Event Structures in Linguistic Form and Interpretation, pages 245-265. Berlin: Mouton de Gruyter.

Bisetto, Antonietta and Chiara Melloni. 2007. Result nominals: a lexicalsemantic investigation. In Proceedings of the Fifth Mediterranean Morphology Meeting.

Dagan, I., O. Glickman, and B. Magnini. 2006. The PASCAL recognising textual entailment challenge. Machine Learning Challenges. Lecture Notes in Computer Science 3944:177-190.

Dixon, Robert M.W. 1991. A new Approach to English Grammar, on Semantic Principles. Oxford: Oxford University Press.

Dowty, David R. 1979. Word meaning and Montague grammar: The semantics of verbs and times in generative semantics and in Montague's PTQ, vol. 7. Berlin: Springer.

Dowty, David R. 1991. Thematic proto-roles and argument selection. Language pages 547-619.

Harel, David, Dexter Kozen, and Jerzy Tiuyn. 2000. Dynamic Logic. Cambridge, MA: The MIT Press, 1st edn.

Hay, Jennifer, Christopher Kennedy, and Beth Levin. 1999. Scalar structure underlies telicity in" degree achievements". In Proceedings of SALT, vol. 9, pages 127-144.

Jackendoff, Ray. 1990. Semantic structures. Cambridge, MA: The MIT press.

Jezek, Elisabetta. 2014. Classes of creation verbs. In R. Simone and F. Masini, eds., Word Classes: Nature, Typology and Computational Representations. Benjamins.

Kennedy, Chris. 2009. Aspectual composition and scalar change. In Workshop on Scalarity and Event Structure.

Kilgarriff, Adam, Vít Baisa, Jan Bušta, Miloš Jakubíček, Vojtěch Kováŕ, Jan Michelfeit, Pavel Rychlỳ, and Vít Suchomel. 2014. The sketch engine: ten years on. Lexicography 1(1):7-36.

Levin, Beth. 1993. English verb classes and alternations: A preliminary investigation. University of Chicago press.

Levin, Beth and Malka Rappaport Hovav. 2010. Lexicalized scales and verbs of scalar change. In Proceedings of the 46th Annual Meeting of the Chicago Linguistics Society, pages 8-10.

McKeon, Richard. 1968. The Basic Works of Aristotle. Random House, New York.

Naumann, Ralf. 2001. Aspects of changes: a dynamic event semantics. Journal of Semantics (18):27-81. 
Piñón, Christopher. 2008. Verbs of creation. In T. H.-Z. Johannes Dölling and M. Schäfer, eds., Event Structures in Linguistic Form and Interpretation, pages 493-521. Berlin - New York: Berlin: Mouton de Gruyter.

Pustejovsky, James. 1995. The Generative Lexicon. Cambridge, MA: Cambridge, MA: The MIT Press.

Pustejovsky, James. 2000. Events and the semantics of opposition. In C. Tenny and J. Pustejovsky, eds., Events as Grammatical Objects, pages 445-482. Stanford, CA: Stanford, CA: CSLI Publications.

Pustejovsky, James. 2013. Dynamic event structure and habitat theory. In Proceedings of the 6th International Conference on Generative Approaches to the Lexicon.

Pustejovsky, James and Elisabetta Jezek. 2011. Scale-shifting and compositionality. In Proceedings of the Workshop on Scalarity in Verb-Based Constructions.

Pustejovsky, James and Jessica Moszkowicz. 2011. The qualitative spatial dynamics of motion. Spatial Cognition and Computation 11(1):15-44.

Pustejovsky, James, Martha Palmer, Annie Zaenen, and Susan Brown. 2016. Integrating Verbnet and GL predicative structures. In Proceedings of the 12th Joint ACL-ISO Workshop on Interoperable Semantic Annotation.

Rappaport Hovav, Malka. 2008. Lexicalized meaning and the internal temporal structure of events. In S. Rothstein, ed., Crosslinguistic and Theoretical Approaches to the Semantics of Aspect, pages 13-42. Benjamins.

Resnik, Philip. 1996. Selectional constraints: An information-theoretic model and its computational realization. Cognition 61(1):127-159.

Suppes, Krantz D. M. Luce R. D., P. and A. Tversky. 1989. Foundations of Measurement Vol. II: Geometrical threshold, and probabilistic representations. Academic Press.

Tenny, Carol L. 1994. Aspectual roles and the syntax-semantics interface. Berlin: Springer.

von Stechow, Arnim. 2001. Temporally opaque arguments in verbs of creation. In C. Cecchetto, ed., Semantic Interfaces: Reference, Anaphora, and Aspect, pages 278-319. Stanford, CA: CSLI Publications. 